\title{
Modulation of pain ratings by expectation and uncertainty: Behavioral characteristics and anticipatory neural correlates
}

\author{
Christopher A. Brown ${ }^{\mathrm{a}, *}$, Ben Seymour ${ }^{\mathrm{b}}$, Yvonne Boyle ${ }^{\mathrm{a}}$, \\ Wael El-Deredy ${ }^{\mathrm{c}}$, Anthony K.P. Jones ${ }^{\mathrm{a}}$ \\ ${ }^{a}$ Human Pain Research Group, Clinical Sciences Building, Hope Hospital, Salford M6 8HD, United Kingdom \\ ${ }^{\mathrm{b}}$ Wellcome Department of Imaging Neuroscience, Functional Imaging Laboratory, 12 Queen Square, London WC1N 3BG, United Kingdom \\ ${ }^{\mathrm{c}}$ School of Psychological Sciences, University of Manchester, Zochonis Building, Oxford Road, Manchester M13 9PL, United Kingdom
}

Received 8 January 2007; received in revised form 30 May 2007; accepted 31 May 2007

\begin{abstract}
Expectations about the magnitude of impending pain exert a substantial effect on subsequent perception. However, the neural mechanisms that underlie the predictive processes that modulate pain are poorly understood. In a combined behavioral and high-density electrophysiological study we measured anticipatory neural responses to heat stimuli to determine how predictions of pain intensity, and certainty about those predictions, modulate brain activity and subjective pain ratings. Prior to receiving randomized laser heat stimuli at different intensities (low, medium or high) subjects $(n=15)$ viewed cues that either accurately informed them of forthcoming intensity (certain expectation) or not (uncertain expectation). Pain ratings were biased towards prior expectations of either high or low intensity. Anticipatory neural responses increased with expectations of painful vs. non-painful heat intensity, suggesting the presence of neural responses that represent predicted heat stimulus intensity. These anticipatory responses also correlated with the amplitude of the Laser-Evoked Potential (LEP) response to painful stimuli when the intensity was predictable. Source analysis (LORETA) revealed that uncertainty about expected heat intensity involves an anticipatory cortical network commonly associated with attention (left dorsolateral prefrontal, posterior cingulate and bilateral inferior parietal cortices). Relative certainty, however, involves cortical areas previously associated with semantic and prospective memory (left inferior frontal and inferior temporal cortex, and right anterior prefrontal cortex). This suggests that biasing of pain reports and LEPs by expectation involves temporally precise activity in specific cortical networks.
\end{abstract}

(C) 2007 International Association for the Study of Pain. Published by Elsevier B.V. All rights reserved.

Keywords: Anticipation; Expectancy; Nociception; Prediction; Inference

\section{Introduction}

Pain expectancy has a strong influence on pain perception (Price, 1999; Fields, 2000; Wager, 2005; Vase et al., 2005). This is of clinical importance since the underlying mechanisms of expectancy have been proposed to play a central role not only in placebo analgesic and nocebo responses (Fields and Price, 2005; Wager, 2005), but also

\footnotetext{
${ }^{*}$ Corresponding author. Tel.: +4401612064528.

E-mail address: Christopher.Brown@Manchester.ac.uk (C.A. Brown).
}

in disability in patients with chronic pain (Crombez et al., 1998; Boersma and Linton, 2006). Modification of beliefs and expectations about pain is one of the primary goals of Cognitive-Behavioral Therapy (CBT), as some chronic pain disorders are thought to be potentiated by rigorously held negative expectations (Vlaeyen and Linton, 2000). However, the neural processes that mediate the impact of expectations on pain perception and behavior are currently poorly understood.

According to theoretical models of attention and perception, the balance of reliance on prior expectation over sensory evidence is critically governed by uncer- 
tainty in those expectations ( $\mathrm{Yu}$ and Dayan, 2005), such that sensory evidence has more influence when expectations are more uncertain. However, recent thinking has suggested that uncertainty necessarily increases pain perception (Ploghaus et al., 2003). Here, we set out to resolve these competing views by investigating the influence of uncertainty on pain ratings across a range of heat intensities, as well as probing the neurophysiological basis for this influence.

We aimed to measure the specific 'top-down' neural correlates of expectation by temporally dissociating pain anticipation from experience. Anticipatory activity toward pain has been investigated with event-related functional Magnetic Resonance Imaging (fMRI) (Ploghaus et al., 1999; Porro et al., 2002, 2003; Wager et al., 2004; Koyama et al., 2005), showing activity in areas commonly associated with nociception (including anterior cingulate (ACC), insular and bilateral inferior parietal cortices). However, fMRI methodologies suffer from the limitation that the haemodynamic response allows only crude disambiguation of neural processes that take place within a few hundred milliseconds before pain onset from those related to stimulus processing, although earlier anticipatory processes can be resolved.

Thus, electroencephalography (EEG) may be better placed to accurately separate pain anticipation from pain experience due to its high temporal resolution. The best characterized anticipatory waveform, the Contingent Negative Variation (CNV), is generated prior to a motor response and is associated with distinct 'early' and 'late' phases with differential activity in the ACC, posterior cingulate, inferior parietal cortex, thalamus, Supplementary Motor Area (SMA) and cerebellum (Cui et al., 2000; Gomez et al., 2001; Gomez et al., 2003; Nagai et al., 2004). In the absence of a required motor response, a Stimulus-Preceding Negativity (SPN) is still observed when anticipating pain, the late phase of which has also been localized to the ACC (Bocker et al., 2001). However, it is not known how the SPN is modulated by uncertainty when anticipating pain.

We therefore designed an experimental paradigm to measure the EEG correlates of early and late phases of pain anticipation estimated using tomographic source localization (LORETA), and the modulation of this activity by certain and uncertain expectations of painful and non-painful heat intensities.

\section{Materials and methods}

\subsection{Subjects}

Fifteen healthy, right-handed subjects, free of psychiatric, neurological, cardiovascular or autonomic disorders, participated in the study (mean age $47 \pm 6.6$ ). Subjects gave informed written consent, and the study was approved by Oldham Local Research Ethics Committee.

\subsection{Experimental procedure}

Laser heat stimuli of $150 \mathrm{~ms}$ duration and a beam diameter of $15 \mathrm{~mm}$ were applied to the dorsal surface of the subjects' right forearm using a $\mathrm{CO}_{2}$ laser stimulator. Between stimuli, the laser was moved randomly over an area $3 \times 5 \mathrm{~cm}$ to avoid habituation, sensitization or skin damage. Subjects wore protective laser safety goggles during the experiment.

An initial psychophysics procedure was performed using a 0-10 sensory rating scale, which was anchored such that a level 4 indicated pain threshold. A ramping procedure was repeated three times to determine three intensities of laser stimulus for each subject: level 3, level 5 and level 7, corresponding to 'low', 'medium' and 'high' intensities of the laser heat stimulus, respectively. These semantic labels were used in the main part of the study to represent the three levels of stimulus intensity. Subjects, however, were not explicitly informed that 'low' corresponded to a level 3, 'medium' to a level 5 and 'high' to a level 7. We then tested that subjects were rating each level of intensity on the numerical scale as expected by giving them a number of randomized pulses at the three intensities, and adjusted the intensity levels to achieve the appropriate sensation if necessary.

On each trial of the experiment (see Fig. 1), a laser stimulus was delivered to the subject every $10 \mathrm{~s}$. A pre-determined randomized sequence of stimulus intensities was used, such that from the subject's perspective each of the 'low' 'medium' and 'high' intensity stimuli, which occurred with equal frequency, could have occurred with equal probability on each trial. Laser stimuli were preceded by the appearance of a visual anticipation cue, occurring $3 \mathrm{~s}$ prior to the laser stimulus, displayed on a computer monitor in front of the subject. The anticipatory cue was a word ('low', 'medium', 'high' or 'unknown') that accurately predicted whether the following laser stimulus was to be a 'low', 'medium' or 'high' intensity ( $50 \%$ of trials, certain expectation), or an 'unknown' pain intensity ( $50 \%$ of trials, uncertain expectation). In total, an equal number of laser stimuli at each intensity level were delivered to induce certain and uncertain expectations. Certain expectations were never violated. The onset of the visual anticipation cue occurred simultaneously with the first of three auditory tones applied using loudspeakers that sounded at once per second until laser stimulus delivery, allowing for accurate prediction in the timing of the laser stimulus. The visual anticipatory cue remained on the computer monitor

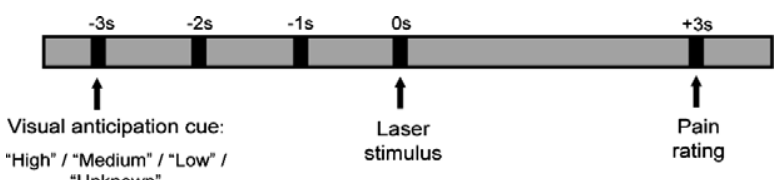

Fig. 1. Experimental design. A visual anticipation cue was presented at $-3 \mathrm{~s}$, indicating to the subjects whether they should expect either 'high', 'medium' or 'low' intensity pain ( $50 \%$ trials) or an 'unknown' intensity of pain $(50 \%$ trials). The visual anticipation cue occurred alongside an auditory tone, and two further auditory tones (at $-2 \mathrm{~s}$ and $-1 \mathrm{~s}$ ) counted down the onset of a $150 \mathrm{~ms}$ laser pulse (at $0 \mathrm{~ms}$ ), in order that subjects could accurately predict the timing of the pain. At $+3 \mathrm{~s}$, an auditory tone indicated to subjects to provide a verbal pain intensity rating, using a $0-10$ pain scale as a reference. 
for the duration of the trial. Three seconds after each laser pulse, an auditory tone of a different quality indicated to the subjects to verbally report the intensity of the experienced pain, using the $0-10$ pain scale. In total, 240 trials were presented in 4 blocks of 60 trials. The experiment lasted approximately $45 \mathrm{~min}$.

\subsection{Electroencephalographic (EEG) recordings}

EEG recordings were taken from 61 scalp electrodes placed according to an extended 10-20 system (Quik-Cap system, Neuroscan, Inc.). Bandpass filters were set at DC $-70 \mathrm{~Hz}$, with a sampling rate of $500 \mathrm{~Hz}$ and gain of 500 . A notch filter was set to $50 \mathrm{~Hz}$ to reduce electrical interference. Electrodes were referenced to the ipsilateral (right) earlobe, and recordings were also taken from the contralateral (left) earlobe for off-line conversion to linked-ears reference. The vertical and horizontal electro-oculograms (EOG) were measured for off-line reduction of blink and eye-movement artifacts.

\subsection{Data analysis}

Behavioral data were analyzed to determine the main effects of, and interactions between, intensity and certainty on pain ratings, using a repeated measures analysis of variance (ANOVA) with certainty (certain, uncertain) and intensity (low, medium, high) as factors. Results were corrected for non-sphericity using Greenhouse-Geisser. Post hoc paired $t$-tests were used to determine the effect of certainty on pain ratings for each intensity individually.

EEG data were analyzed using Neuroscan Edit 4.3. An ocular artifact reduction algorithm (Semlitsch et al., 1986) was performed. The data were epoched into single trials of $5.5 \mathrm{~s}$ duration starting $1000 \mathrm{~ms}$ before the visual anticipation stimulus, including $3000 \mathrm{~ms}$ of anticipation, and ending $1500 \mathrm{~ms} \mathrm{sec-}$ onds after the laser stimulus. Epochs were visually inspected for further ocular artifacts that had escaped automatic removal, and deleted if necessary. Linear trends over the whole epoch were removed using the entire epoch to calculate the linear component in all channels; the whole epoch was used in order to minimize removal of linear trends that may have specifically resulted from the anticipatory neural responses we were intending to measure. Each epoch was then baseline corrected to the $500 \mathrm{~ms}$ interval preceding the visual anticipation cue. The data were then averaged for each intensity level in each condition separately. The three intensity conditions occurring for uncertain expectation were averaged together before analyzing anticipatory responses, leaving four averaged sets of data for each subject (corresponding to the four visual anticipation cues: 'low', 'medium', 'high', 'unknown'). Data were referenced to the common average (i.e. the average electrical activity over all electrodes) before proceeding with data analysis, although ERP waveforms are presented according to the linked-ears reference.

Two $500 \mathrm{~ms}$ phases of the anticipatory brain response were analyzed. An 'early' phase, at -2500 to $-2000 \mathrm{~ms}$ preceding the laser stimulus, was chosen as the earliest part of the anticipatory response that could be measured without interference from visual-evoked responses resulting from the anticipatory cue. A 'late' phase, at -500 to $0 \mathrm{~ms}$ preceding the laser stimulus, was chosen to represent processes taking place in immediate preparation for the impending laser stimulus. We reasoned that these early and late phases would be analogous to the early and late phases of the CNV demonstrated in previous studies (Gomez et al., 2003). It is possible that pre-motor responses such as the readiness potential, thought to contribute to the CNV, may also contribute to the SPN. However, we minimized the effects of motor components in the SPN by delaying each subject's response to the laser stimuli until $3 \mathrm{~s}$ after each stimulus. The SPN as recorded with this study is therefore more associated with anticipation of the sensory perception of pain. However, some motor components may be involved that are associated with task-unrelated motor responses to anticipation of pain (e.g. the need to inhibit arm withdrawal from the pain during the experimental procedure).

For each of the two phases, the mean amplitude of the SPN over $500 \mathrm{~ms}$ was calculated for each electrode and in each condition. The mean amplitude was then averaged across all conditions, and the electrode with the highest amplitude was determined. The mean amplitude of the SPN at this electrode and a number of other representative midline electrodes $(\mathrm{Fz}$, $\mathrm{FCz}, \mathrm{Cz}, \mathrm{CPz}$ and $\mathrm{Pz}$ ) were then compared between the certain conditions (low, medium and high) using a repeated measures ANOVA. Results at significant electrodes were further assessed using paired-samples $t$-tests to compare SPN amplitude for either low vs. medium intensity or medium vs. high intensity stimuli. We also made a comparison between certain and uncertain conditions, averaged across all intensities, for the two phases at these midline electrodes using a repeated measures ANOVA. All ANOVA results were corrected for non-sphericity using Greenhouse-Geisser.

We also analyzed the P2 peak of the Laser-Evoked Potential (LEP). Each subject's P2 peak latency was determined at electrode $\mathrm{Cz}$ where the topography of the P2 peak was maximal. A $20 \mathrm{~ms}$ section of the P2 peak, $10 \mathrm{~ms}$ either side of the latency at which the P2 peak showed maximal amplitude, was averaged for each subject and for each of the six conditions (i.e. high, medium and low during both certain and uncertain expectation). These data were entered into a repeated measures ANOVA $(n=13)$ to explore the main effects and interactions between certainty and intensity on the amplitude of the LEP. The analysis excluded two subjects who did not show a distinct enough P2 peak to allow for individual latency information to be extracted. ANOVA results were corrected for non-sphericity using GreenhouseGeisser. Furthermore, we made a comparison between the P2 LEP peak and the amplitude of the SPN during late anticipation, to determine whether SPN amplitude predicts LEP peak amplitude and whether this effect is dependent on the level of certainty. Firstly, LEP amplitudes for each of the high, medium and low intensity stimuli in the certain condition were correlated with the average SPN amplitude during certain expectation, and likewise for LEP and SPN amplitudes during uncertain expectation. This allowed us to determine whether certainty affected the relationship between the SPN and the LEP. Secondly, LEP amplitudes for each of the high, medium and low intensity stimuli in the certain condition were correlated with the amplitude of the corresponding SPN in that condition, i.e. for each inten- 
sity individually. This allowed us to confirm whether anticipatory effects on LEP amplitude during certainty occurred within each experimental condition.

Cortical sources of the SPN were estimated with low-resolution electromagnetic tomography (LORETA), using the LORETA-KEY software (Pascual-Marqui et al., 2002). The software uses a three-shell spherical head model registered to the Talairach anatomical brain atlas, although the electrode coordinates used were determined from a co-registration between spherical and realistic head geometry that created a best-fit model (Towle et al., 1993). LORETA estimates ERP sources in grey matter volume, including some of the hippocampus and amygdala, to a $7 \mathrm{~mm}^{3}$ grid resolution using the digitized MNI probability atlas (Mazziotta et al., 2001). Time-domain EEG files were converted to current density vector field magnitude using this technique.

There is sizeable evidence to suggest that LORETA is accurate in localizing the sources of ERPs. Research based on the use of simulated data (Cuffin et al., 2001a,b; Yao and Dewald, 2005) suggests that source localization techniques are able to localize activation to within $10-20 \mathrm{~mm}$ (which equates to 1-3 LORETA voxels). Similar estimates have been provided from cross-validation studies of epileptic foci localization (Lantz et al., 2003; Michel et al., 2004), which show strong correspondence with PET data (Zumsteg et al., 2005). There is also substantial similarity between intra-cerebral recordings and the results of source localization (Seeck et al., 1998; TrebuchonDa Fonseca et al., 2005). Moreover, combined EEG/fMRI studies have shown that LORETA localizations are proximal to fMRI localizations to within $14-16 \mathrm{~mm}$ (Mulert et al., 2004; Vitacco et al., 2002).

Statistical maps were created from the LORETA solutions by performing voxel-wise $t$-tests, using a non-parametric randomization and permutation test that requires minimal assumptions for validity (see Nichols and Holmes, 2002 for a detailed description of this test). This also produces a $t$-threshold for significant differences in cluster sizes of activation. The cluster test is performed using 10 pre-determined thresholds that are linearly arranged from the maximum absolute $t$-value (voxel-by-voxel), towards zero (without including zero). Contiguous voxels above each threshold are grouped into clusters, and the most significant value is reported.

Prior to performing $t$-tests, LORETA solutions were intensity normalized in order to eliminate subject-to-subject global variations. LORETA was then used to compute two main comparisons of interest, addressing the sources of anticipatory activity specific firstly to intensity, and secondly to certainty. To identify areas related to the anticipation of intensity, we directly compared expectation of high to low intensity stimuli by voxel-wise $t$-test in the certain expectation condition. This was done both for the early and late phases of the anticipatory response. Secondly, in each phase, the effect of certainty vs. uncertainty on estimated sources was determined using a voxel-wise $t$-test, after averaging each of these conditions across the three intensities.

On a note of caution, our interpretation of the results should take into account the low spatial resolution and the anatomical and physiological assumptions required to analyze EEG data using LORETA. In particular, there may be particular inaccuracy in the localization of subcortical sources.

\section{Results}

\subsection{Behavioral data}

The behavioral data showed that expectations significantly influenced pain ratings: expectations of moderately painful heat ('high' intensity) increased pain ratings, whereas expectations of non-painful heat ('low' intensity) decreased pain ratings. Pain ratings (mean, (SD)) for certain and uncertain expectations, respectively, were: $5.85(0.45)$ and $5.63(0.33)$ for high intensity stimuli; $4.34(0.56)$ and $4.26(0.60)$ for medium intensity stimuli; $2.09(0.73)$ and 2.49 (0.66) for low intensity stimuli. ANOVA results showed a significant effect of intensity $(F(1.2,14.6)=201 ; p<0.000)$, and an interaction between intensity and certainty $(F(2,23.8)=7.81 ; p<0.003) \quad$ (see Fig. 2). Post hoc paired $t$-tests revealed that the interaction between intensity and certainty resulted from reduced pain ratings for low intensity stimuli as a result of certain relative to uncertain expectation $(p=0.001)$, and increased pain ratings for high intensity stimuli as as a result of certain relative to uncertain expectation $(p=0.025)$. In other words certainty increased the rating of high intensity stimuli and reduced the rating of low intensity stimuli. Ratings were slighty lower overall than determined during the psychophysics procedure at the beginning of the study, possibly due to habituation and/or a reduction in anxiety levels.

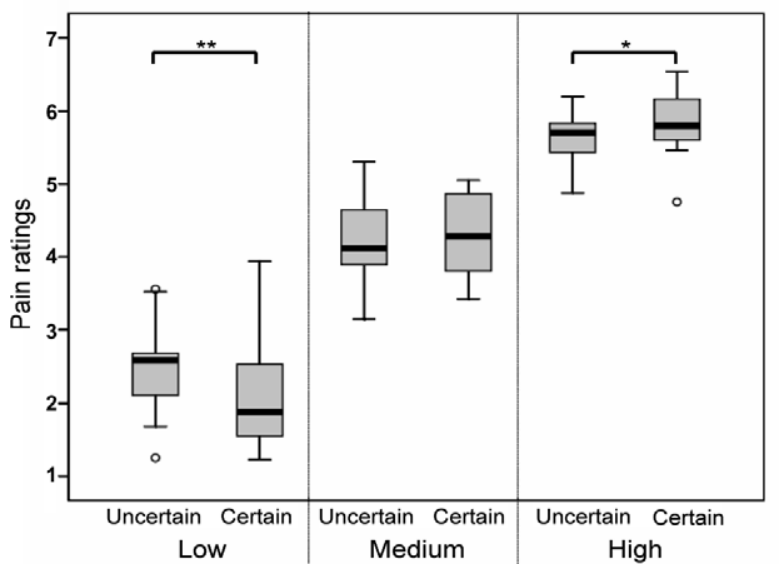

Fig. 2. Box plot showing the median, inter-quartile range and full range of pain ratings across subjects. Low, medium and high intensity pain conditions are shown, comparing uncertain and certain expectation for each. Outliers are shown as small circles. A 0-10 pain scale was used as a reference by the subject, anchored such that level 4 corresponded to pain threshold. An interaction between intensity and certainty (repeated measures ANOVA, $p=0.003$ ), was explained by significantly reduced pain ratings for certain vs. uncertain expectation of low intensity stimuli $\left({ }^{* *} p=0.001\right)$ and significantly increased pain ratings for certain vs. uncertain expectation of high intensity stimuli $\left({ }^{*} p=0.025\right)$. 


\subsection{Stimulus-Preceding Negativity ( $S P N)$ data}

We observed a slow negative wave during anticipation of the pain stimulus, which we refer to as the Stimulus-Preceding Negativity. Topographic maps of the grand average for the mean of all conditions (Fig. 3) show the distribution of the SPN in the early and late phases. In the early phase, the SPN has a broad fronto-central distribution with peak activity at electrode FC3. In the late phase, the SPN has a more central distribution with peak activity at electrode $\mathrm{Cz}$. In the late phase, a repeated measures ANOVA (Fig. 4) of the mean amplitude of the SPN across the certain conditions (low, medium and high) showed that there was significant modulation of the SPN by expected intensity at electrodes $\mathrm{Cz} \quad(F(1.9,27)=3.6 ; \quad p<0.04)$ and $\mathrm{CPz}$ $(F(1.7,22.2)=6.3 ; \quad p<0.009)$. Paired-samples $t$-tests showed that a step-wise increase in heat intensity from low (non-painful) to medium (low pain) was significant at electrode $\mathrm{CPz}(p<0.05)$, but that there was no significant difference between medium and high intensity SPN amplitude at these electrodes, although a qualitative difference is visible in Fig. 4. There was no significant effect of expected intensity on the SPN in the early phase, nor was there a specific effect of certainty relative to uncertainty in either the early or late phases.

\subsection{Laser-Evoked Potential (LEP) data}

Analysis of the P2 LEP showed a main effect of intensity $(p<0.01)$ but did not reveal an effect of certainty, nor an interaction between intensity and certainty. However, certain expectation was found to alter the relationship between the amplitude of the P2 LEP peak and the SPN for painful stimuli. During certain expectation, the mean amplitude of the late SPN averaged over the three intensities predicted the P2 peak amplitude for high $(r=0.59, p<0.03)$ and medium $(r=0.55, p<0.04)$ intensity stimuli (see Fig. 4), but not for low intensity stimuli. During uncertain expectation, the late SPN was not predictive of the P2 LEP peak amplitude for any of high, medium or low intensities. Furthermore, when the amplitude of the SPN was considered for each intensity individually during the certain conditions, SPN amplitude predicted the P2 peak amplitude for high $(r=0.54, p<0.05)$ and medium $(r=0.65, p<0.01)$ intensity stimuli, but not for low intensity stimuli.

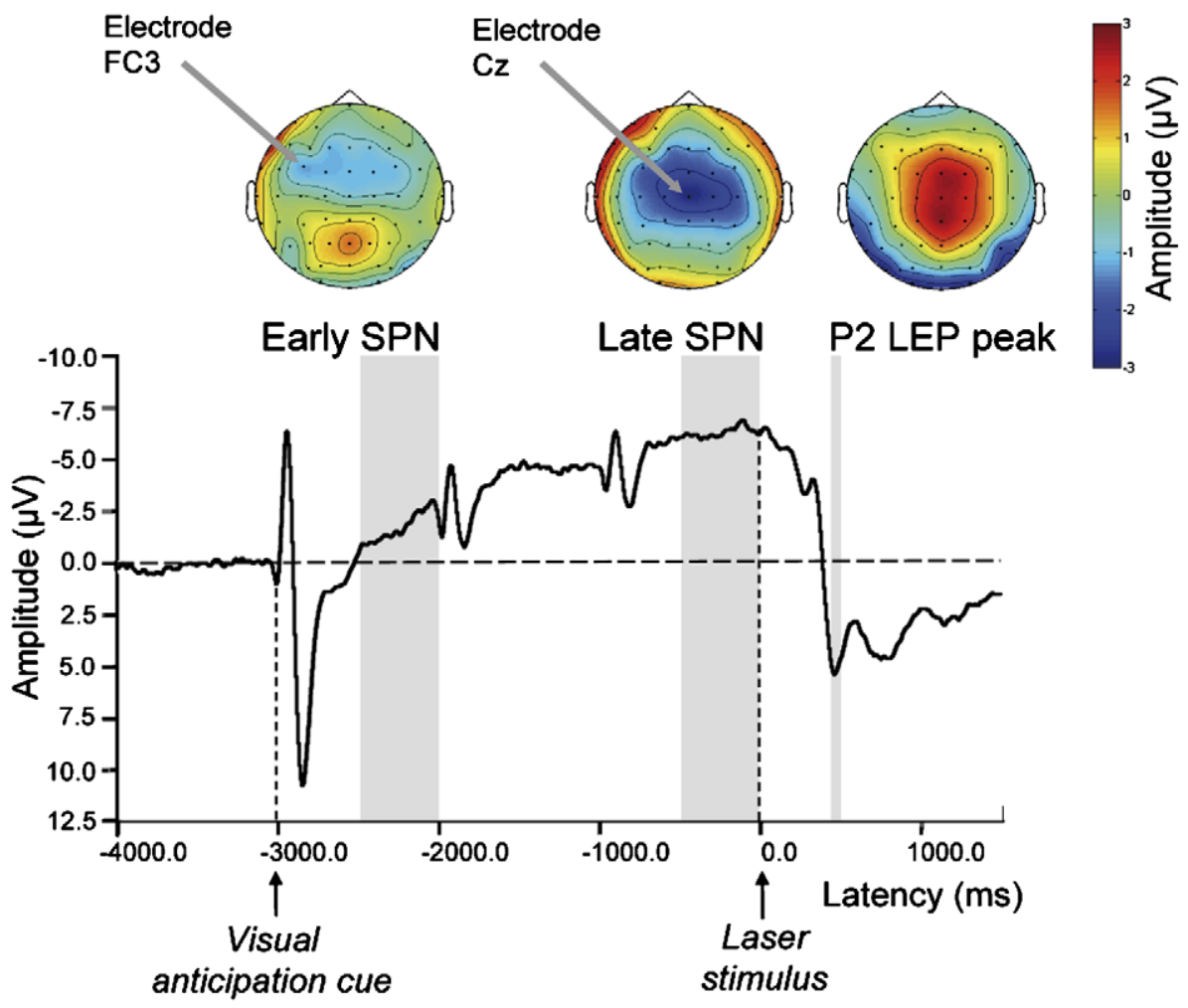

Fig. 3. Grand average across subjects of waveforms and topographic maps over all conditions. The temporal range of early ( -2500 to $-2000 \mathrm{ms)}$ and late ( -500 to $0 \mathrm{~ms})$ periods of the Stimulus-Preceding Negativity (SPN) is shown $(n=15)$, in addition to the P2 peak of the post-stimulus LaserEvoked Potential (LEP) $(n=13)$. The SPN is the gradually increasing negative potential occurring between $-3000 \mathrm{~ms}$ and $0 \mathrm{~ms}$. In the early phase, the SPN showed a broad fronto-central distribution with peak activity at electrode FC3. In the late phase, the SPN showed a more central distribution with peak activity at electrode Cz. The P2 peak of the LEP is also maximal as electrode Cz. Also evident are visual-evoked potentials caused by the visual anticipation cue, auditory-evoked potentials caused by predictive auditory tones. Waveforms are presented according to a linked-ears reference, whereas topographic maps are presented with respect to a common average reference. 

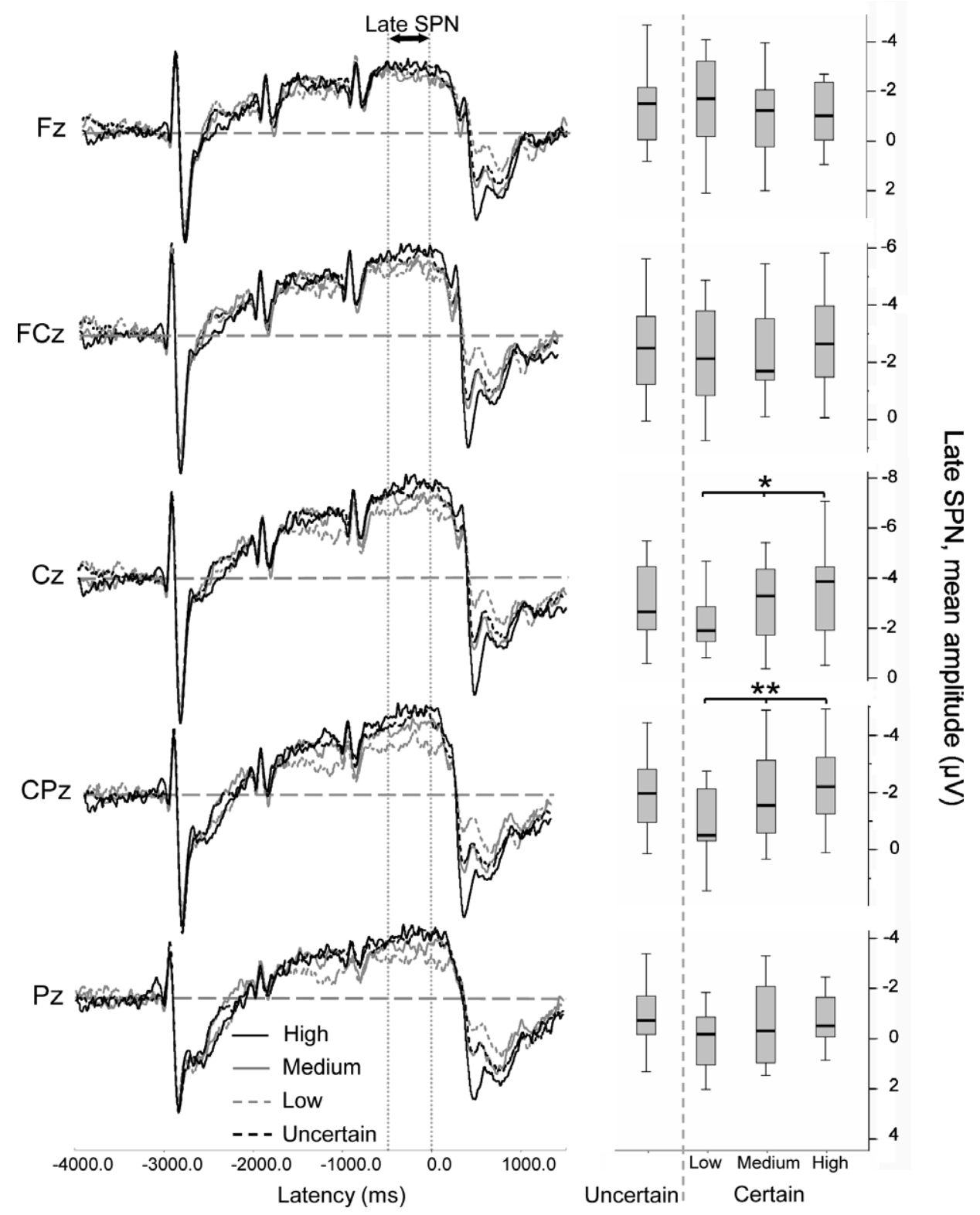

Fig. 4. Grand average $(n=15)$ waveforms over the course of the experimental trial for certain expectation (of high, medium and low pain) and uncertain expectation at representative electrodes. The mean amplitude across the $500 \mathrm{~ms}$ time period of the late phase of the SPN for each condition is shown as a box plot for each electrode. During certain expectation, the amplitude of the SPN during the late phase was significantly modulated by the intensity of expected pain at electrodes $\mathrm{Cz}\left({ }^{*} p=0.04\right)$ and $\mathrm{CPz}\left({ }^{* *} p=0.009\right)$. Displayed waveforms are referenced to linked-ears.

\subsection{Source analysis (LORETA) of the SPN}

Significant changes in current density were found using LORETA during pain anticipation for the two main comparisons of interest (see Table 1).

In the first LORETA comparison, in which certain expectation of high intensity stimuli was compared to certain expectation of low intensity stimuli, we did not observe any statistically significant results at our threshold of significance, in either early or late anticipatory phases (see Fig. 5).

The second LORETA comparison, in which certain expectation was contrasted with uncertain expectation
(Fig. 6), identified regions of the brain that were specifically modulated by certainty in each of the early and late phases. In the early phase (Fig. 6a), uncertain expectation was associated with increased clusters in the left middle frontal gyrus (referred to as dorsolateral prefrontal cortex, DLPFC), posterior cingulate cortex (PCC), left (contralateral) inferior parietal cortex (IPL) and right superior frontal gyrus (SFG). In the late phase (Fig. 6b), uncertainty was associated with greater activity in voxels in the right IPL and right SFG.

For the opposite contrast (i.e. certain $>$ uncertain), in the early phase certain expectation was associated with increased clusters of activity in the right superior frontal 
Table 1

Brain regions showing significant differences in current density when comparing certain and uncertain expectations

\begin{tabular}{lll}
\hline Region & Area & $\begin{array}{l}\text { Talairach } \\
\text { coordinates (not } \\
\text { stated for cluster } \\
\text { statistics) }\end{array}$ \\
\cline { 2 - 3 } & $x \quad y \quad z$ & $z$ \\
& &
\end{tabular}

\section{Early certain $>$ uncertain}

$\begin{array}{lll}\text { Fusiform gyrus }^{\mathrm{a}} & \text { L } & 20\end{array}$

Sub-gyral temporal lobe ${ }^{\mathrm{a}} \quad$ L $\quad 21$

Inferior temporal gyrus $^{\text {a }} \quad$ L 37

Inferior frontal gyrus $^{\mathrm{a}} \quad \mathrm{L} \quad 10 / 47$

Subcallosal gyrus $^{\text {a }} \quad$ L $34 / 25$

Hippocampus $^{\mathrm{a}}$

Superior frontal gyrus ${ }^{\mathrm{a}}$

Uncus $^{\mathrm{a}}$

Medial frontal gyrus ${ }^{\mathrm{a}}$

Early uncertain $>$ certain

Middle frontal gyrus ${ }^{\mathrm{a}}$

Cingulate gyrus ${ }^{\mathrm{a}}$

Inferior parietal lobule ${ }^{a}$

Superior frontal gyrus ${ }^{\mathrm{a}}$

Insula $^{\text {a }}$

Postcentral gyrus ${ }^{\mathrm{a}}$

Precuneus $^{\mathrm{a}}$

Middle occipital gyrus ${ }^{\mathrm{a}}$

Precentral gyrus ${ }^{\mathrm{a}}$

Late uncertain $>$ certain

Inferior parietal lobule $\mathrm{e}^{\mathrm{a}, \mathrm{b}}$

\begin{tabular}{llllll} 
Superior frontal gyrus ${ }^{\mathrm{a}, \mathrm{b}}$ & $\mathrm{R}$ & 6 & 18 & 03 & 64 \\
\hline
\end{tabular}

${ }^{a}$ Brain regions showing significant differences $(p<0.05)$ in cluster size. Note that significant results from the cluster test cannot be associated with a specific voxel, and therefore talairach coordinates are not provided.

${ }^{\mathrm{b}}$ Brain regions showing significant differences $(p<0.05)$ in current density within a particular voxel, quoted here in talairach coordinates. $\mathrm{L}$, left hemisphere activation; $\mathrm{R}$, right hemisphere activation; $\mathrm{B}$, bilateral activation.

gyrus (referred to as anterior prefrontal cortex, aPFC in Fig. 6a). Certain expectation was also associated with increased cluster size in left temporal areas, including fusiform gyrus and left inferior frontal gyrus (IFG). Further increases in cluster size centered around the medial temporal lobes (including an area encompassing the hippocampus) and subcallosal cingulate gyrus (which we refer to as subgenual anterior cingulate cortex, sACC).

\section{Discussion}

Our data illustrate four key findings. First, expectations bias ratings of heat stimuli toward the predicted outcome, and in the direction of both higher and lower intensities. Second, anticipatory EEG activity varies according to the predicted magnitude of the anticipated heat intensity. Third, the amplitude of the anticipatory response predicts the amplitude of the pain-evoked response during certain expectation but not uncertain expectation. Fourthly, distinct cortical networks are associated with certain vs. uncertain expectation.

\subsection{Behavioral effects of expectation and uncertainty}

Our behavioral data cast light on how the perception of heat intensity might be modulated by uncertainty in expectations about pain. The uncertain condition incorporated two distinct types of uncertainty: true uncertainty, in which the subjects had no knowledge of the outcome probabilities, and unreliability, which gradually replaced the former as the subject came to learn the outcome distribution, namely that the 'unknown' condition was an inherently poor predictor of the future pain. This latter probability distribution is approximately centered on medium intensity stimuli (since the high, medium and low intensities were equi-probable), and our data suggest the possibility of a perceptual bias towards this in the uncertain condition, relative to the certain condition. Our results are consistent with previous data (Arntz et al., 1991; Wager et al., 2004; Lorenz et al., 2005), and with contemporary statistical (e.g. Bayesian) models of perception. Broadly stated, these models suggest that prior expectation is balanced with the current sensory evidence in order to make a statistically optimal inference about some property, such as the intensity, of a stimulus. Indeed, such models offer a pervasive account of perceptual inference across a number of sensory modalities (Rao et al., 2002; Kersten and Yuille, 2003; Friston, 2005; Wager, 2005). Although our experiment does not directly test a Bayesian model of perception (which is one amongst a number of plausible theoretical models for combining prior expectations and sensory input), this theoretical framework provides an elegant model for interpreting the current data.

It is noted that any study involving explicit predictions and subjective reports can never exclude intrusion of subject report biases. Although the time period between the pain stimulus and the verbal report was short (3s), suggesting that an influence on retrospective memory ought to be relatively minimal, any explicit prediction task is potentially open to conformability biases (i.e. subjects report what they think they ought to feel, rather than what they actually do feel). In theory, uncertainty can be manipulated purely implicitly in pavlovian designs, although exerting experimental control over the selective acquisition and expression of implicit but not explicit predictions of pain in such tasks is likely to be extremely challenging. However, setting aside the inescapable superposition of conformability biases, one should reassert that the mechanisms by which explicit information modulates pain are of substantial interest. 
a
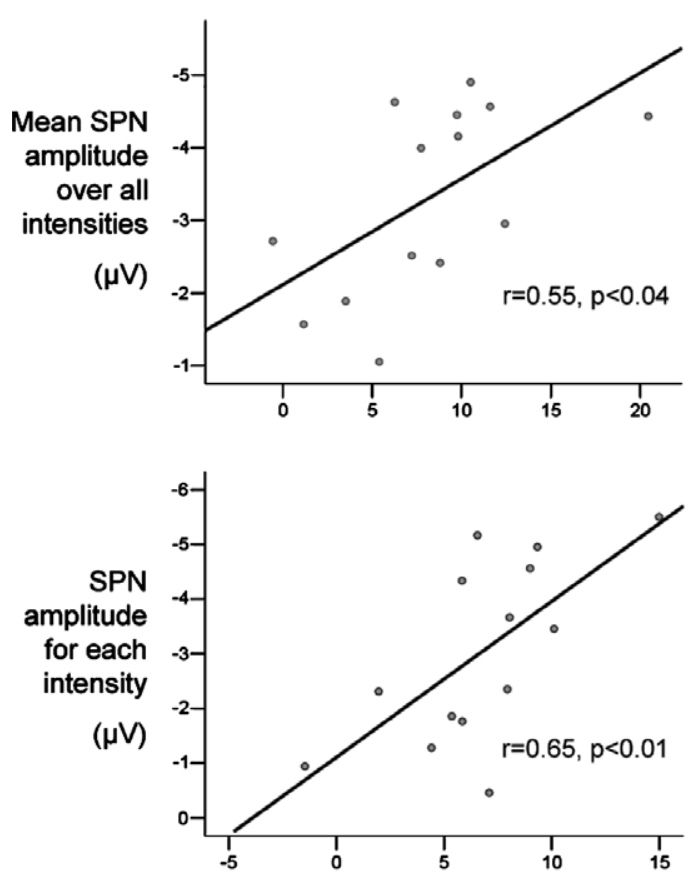

\section{b Certain expectation, high intensity}
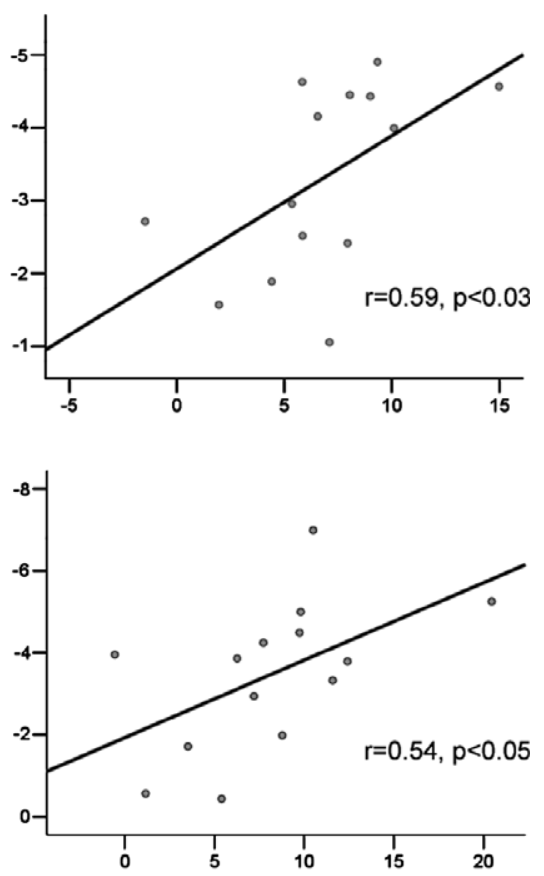

\section{P2 amplitude $(\mu \mathrm{V})$}

Fig. 5. Correlations of SPN amplitude with P2 LEP peak amplitude. For (a) medium intensity stimuli and (b) high intensity stimuli, the P2 LEP peak amplitude correlated $(n=13)$ with both the mean SPN amplitude over all intensities in the certain condition, and the SPN amplitude for each individual intensity condition.
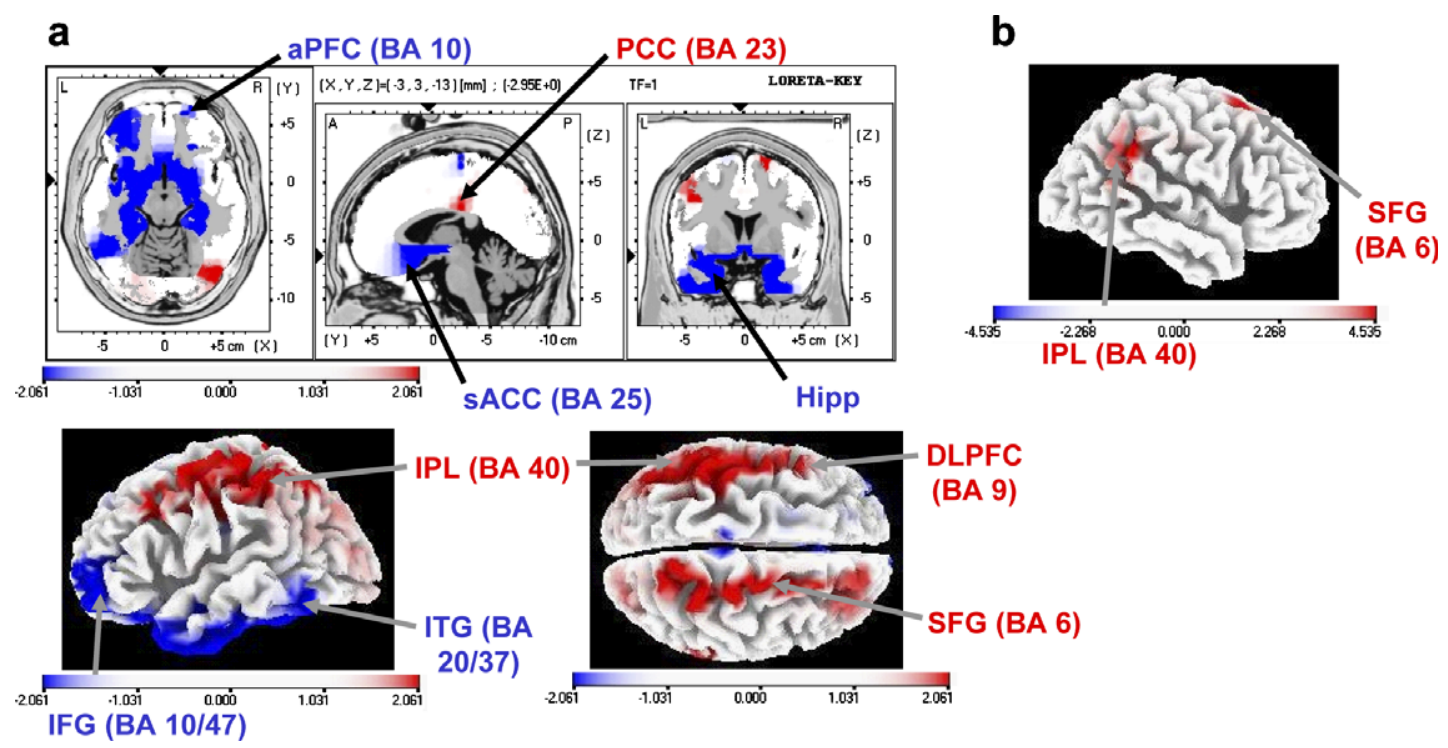

Fig. 6. Comparison of sources of the SPN during certain expectation relative to uncertain expectation estimated with LORETA. (a) Early anticipatory phase: certain vs. uncertain expectation (brain regions in blue) was associated with increased clusters in the medial temporal lobe (including the hippocampus, Hipp), subgenual anterior cingulate cortex (sACC, BA 25), left inferior frontal gyrus (IFG, BA 10/47) and left inferior temporal and fusiform areas (ITG, BA 20/37). Uncertain vs. certain expectation (brain regions in red) was associated with increased clusters in left dorsolateral prefrontal cortex (DLPFC, BA9), superior frontal gyrus (SFG, BA 6/9), inferior parietal lobule (IPL, BA 40) and posterior cingulate cortex (PCC, BA 23). (b) Late anticipatory phase: uncertain vs. certain expectation (brain regions in red) was associated with increased current density in the right inferior parietal lobule (IPL, BA 40) and right superior frontal gyrus (SFG, BA 6). 
4.2. Neural responses during anticipation of different heat intensities

Our EEG data demonstrate coding of heat stimulus intensity just prior to the onset of the stimuli, but not during the early anticipatory phase. Although our ANOVA results did not reveal an effect of certainty on the pain-evoked response (LEPs), we did find that the amplitude of LEPs for painful (but non-painful) stimuli was predicted by late anticipatory activity during certain (but not uncertain) expectation. Early and late phases of anticipatory activity may reflect distinct processes in pain expectancy. Earlier responses may reflect motivational processes, since these typically occur at the time when expectation is first generated (Seymour et al., 2004). However, late phase anticipatory responses would seem much more suited to underlie the 'topdown' perceptual influence, or predictive coding (Rao and Ballard, 1999), of expectancy-related information on ascending nociceptive input.

The LORETA of this late phase of the anticipatory response failed to identify sources modulated by expected intensity. This may be because this is encoded too diffusely, or located at multiple sources, to enable source identification with the currently available statistical tools. Clearly this is an important goal for future studies.

\subsection{Cortical areas mediating certainty and uncertainty}

The contrast of the SPN between the certain and uncertain conditions, across all intensities of heat stimulus (which were exactly balanced), did not reveal any significant differences in the midline electrodes analyzed. However, we were able to identify dissociable brain regions using source analysis. The uncertain condition (relative to the certain condition) was associated with a shift in activity towards more superior and lateral cortical areas (left DLPFC, PCC, bilateral IPL, and right SFG). Greater areas of activation in the certain condition (relative to the uncertain condition) included aPFC, inferior frontal, temporal and subgenual cingulate cortices. In addition, sources of activity that included the hippocampus were identified. However, we consider it unsafe to make inferences from scalp ERPs about subcortical and deep cortical regions; hence, we focus our discussion on the more superficial cortical sources found.

Given that our behavioral data showed no main effect of certainty on pain ratings across the three intensities, these brain networks are unlikely to be a reflection of differences in perceived intensity. Contemporary ideas about how the brain deals with uncertainty are deeply intertwined with theories of attention and learning (Dayan et al., 2000). Cues that harbor uncertain predictions stimulate attention and augment learning, both in a motivational sense (to direct optimal action), and a representational sense (to allow optimal perceptual inference). Consistent with this idea, the brain areas more involved during uncertainty form part of a well-established fronto-parietal network typically associated with attentional function, including ego-spatial, somatosensory and pain functions (Posner and Petersen, 1990; Coull, 1998; Carlsson et al., 2000; Corbetta and Shulman, 2002; Macaluso and Driver, 2003; Behrmann et al., 2004). Voluntary attentional shifts towards pain specifically activate a selective attention network involving DLPFC and IPL (Peyron et al., 1999). In the present study, the left IPL was associated with uncertainty in the early phase, whereas the right IPL was associated with uncertainty in the late phase. The left IPL has been associated with temporal attention to non-painful stimuli (Coull and Nobre, 1998), whereas the right IPL has been associated with spatial attention to both non-painful (Coull and Nobre, 1998) and painful (Kulkarni et al., 2005) stimuli.

The contrast between the certain and uncertain expectation conditions may reflect memory processing associated with the predictive cues. Left IFG and left temporal gyrus activations during certain expectation are consistent with results from studies showing explicit memory processing in response to semantic cues in these areas (Wiggs et al., 1999; Gerlach et al., 2002). We also observed increased activation of the anterior prefrontal cortex (aPFC) in the region of the frontal pole during greater certainty. The function of the aPFC appears to have complex integrative functions (Koechlin et al., 2003), but the specific area identified in the present study is required for prospective memory, i.e. memory for intentions that will be realized in the future (Burgess et al., 2001; Okuda et al., 2003). This might suggest a possible role in mediating mnemonic influences on future behavior, consistent with the influence of predictive cues on pain ratings, although this remains speculative.

\subsection{Clinical implications}

Placebo and nocebo responses are partly mediated by simple cue-outcome contingencies but are particularly significant when induced by verbal expectations (Benedetti et al., 2003; Fields and Price, 2005; Watson et al., 2006). Here, we show that predictive verbal information can both increase and decrease the perception of pain by manipulating the statistical properties of the predictions, namely the uncertainty. Thus, our data are consistent with the possibility that one component of placebo and nocebo effects might be a result of the brain's integration of prior expectancy and current sensory information to make an optimal perceptual inference. 
The therapeutic effects of Cognitive-Behavioral Therapy on pain perception may be mediated by increasing uncertainty about rigorously held negative expectations. Consistent with our source analysis data during uncertainty, attention to sensory evidence may then become a more important resource for informing behavior, thereby maximising adaptivity to pain. Indeed, behavioral change is thought to be more successful using experience-based therapeutic methods such as exposure (McCracken, 2005), and also attentional training (Morley et al., 2004; McCracken, 2005) which minimizes cognitive elaboration of prior expectations and increases sensory awareness (Bishop et al., 2004). Indeed, focusing attention on the sensory aspects of pain can increase pain tolerance and recovery from pain in healthy subjects and chronic pain patients (McCaul and Haugtvedt, 1982; Cioffi and Holloway, 1993; Keogh et al., 2000; Michael and Burns, 2004).

\section{Conclusions}

The pattern of modulation of pain ratings by expectation is consistent with models of perceptual inference. We present evidence that neural responses during anticipation of pain may code for the predictive information required to modulate pain perception, and that uncertain predictions may reduce this modulation via activation of a network associated with attention and learning. These findings underscore the importance of investigating the mechanisms of prior expectancy in the pathophysiology of chronic pain.

\section{Acknowledgements}

This work was funded by the University of Manchester and the Arthritis Research Campaign. We thank Professor Steve Williams and Dr. Lloyd Gregory at the University of Manchester for their input and support of this work.

\section{References}

Arntz A, van den Hout MA, van den BG, Meijboom A. The effects of incorrect pain expectations on acquired fear and pain responses. Behav Res Ther 1991;29:547-60.

Behrmann M, Geng JJ, Shomstein S. Parietal cortex and attention. Curr Opin Neurobiol 2004;14:212-7.

Benedetti F, Pollo A, Lopiano L, Lanotte M, Vighetti S, Rainero I. Conscious expectation and unconscious conditioning in analgesic, motor, and hormonal placebo/nocebo responses. J Neurosci 2003;23:4315-23.

Bishop SR, Lau M, Shapiro S, Carlson L, Anderson ND, Carmody J, et al. Mindfulness: a proposed operational definition. Clin Psychol 2004;11:230-41.

Bocker KB, Baas JM, Kenemans JL, Verbaten MN. Stimuluspreceding negativity induced by fear: a manifestation of affective anticipation. Int J Psychophysiol 2001;43:77-90.
Boersma K, Linton SJ. Expectancy, fear and pain in the prediction of chronic pain and disability: a prospective analysis. Eur J Pain 2006; 10:551-7.

Burgess PW, Quayle A, Frith CD. Brain regions involved in prospective memory as determined by positron emission tomography. Neuropsychologia 2001;39:545-55.

Carlsson K, Petrovic P, Skare S, Petersson KM, Ingvar M. Tickling expectations: neural processing in anticipation of a sensory stimulus. J Cogn Neurosci 2000;12:691-703.

Cioffi D, Holloway J. Delayed costs of suppressed pain. J Pers Soc Psychol 1993;64:274-82.

Corbetta M, Shulman GL. Control of goal-directed and stimulusdriven attention in the brain. Nat Rev Neurosci 2002;3:201-15.

Coull JT. Neural correlates of attention and arousal: insights from electrophysiology, functional neuroimaging and psychopharmacology. Prog Neurobiol 1998;55:343-61.

Coull JT, Nobre AC. Where and when to pay attention: the neural systems for directing attention to spatial locations and to time intervals as revealed by both PET and fMRI. J Neurosci 1998;18:7426-35.

Crombez G, Eccleston C, Baeyens F, Eelen P. When somatic information threatens, catastrophic thinking enhances attentional interference. Pain 1998;75:187-98.

Cuffin BN, Schomer DL, Ives JR, Blume H. Experimental tests of EEG source localization accuracy in spherical head models. Clin Neurophysiol 2001a;112:46-51.

Cuffin BN, Schomer DL, Ives JR, Blume H. Experimental tests of EEG source localization accuracy in realistically shaped head models. Clin Neurophysiol 2001b;112:2288-92.

Cui RQ, Egkher A, Huter D, Lang W, Lindinger G, Deecke L. High resolution spatiotemporal analysis of the contingent negative variation in simple or complex motor tasks and a non-motor task. Clin Neurophysiol 2000;111:1847-59.

Dayan P, Kakade S, Montague PR. Learning and selective attention. Nat Neurosci 2000;3:1218-23.

Fields HL. Pain modulation: expectation, opioid analgesia and virtual pain. Prog Brain Res 2000;122:245-53.

Fields HL, Price DD. Placebo analgesia. In: Wall PD, Melzack R, editors. Textbook of pain Churchill Livingstone, 2005.

Friston KJ. A theory of cortical responses. Philos Trans R Soc Lond B Biol Sci 2005;360:815-36.

Gerlach C, Aaside CT, Humphreys GW, Gade A, Paulson OB, Law I. Brain activity related to integrative processes in visual object recognition: bottom-up integration and the modulatory influence of stored knowledge. Neuropsychologia 2002;40:1254-67.

Gomez CM, Delinte A, Vaquero E, Cardoso MJ, Vazquez M, Crommelinck M, et al. Current source density analysis of $\mathrm{CNV}$ during temporal gap paradigm. Brain Topogr 2001;13:149-59.

Gomez CM, Marco J, Grau C. Preparatory visuo-motor cortical network of the contingent negative variation estimated by current density. Neuroimage 2003;20:216-24.

Keogh E, Hatton K, Ellery D. Avoidance versus focused attention and the perception of pain: differential effects for men and women. Pain 2000;85:225-30.

Kersten D, Yuille A. Bayesian models of object perception. Curr Opin Neurobiol 2003;13:150-8.

Koechlin E, Ody C, Kouneiher F. The architecture of cognitive control in the human prefrontal cortex. Science 2003;302:1181-5.

Koyama T, McHaffie JG, Laurienti PJ, Coghill RC. The subjective experience of pain: where expectations become reality. Proc Natl Acad Sci USA 2005;102:12950-5.

Kulkarni B, Bentley DE, Elliott R, Youell P, Watson A, Derbyshire $\mathrm{SW}$, et al. Attention to pain localization and unpleasantness discriminates the functions of the medial and lateral pain systems. Eur J Neurosci 2005;21:3133-42.

Lantz G, Grave dP, Spinelli L, Seeck M, Michel CM. Epileptic source localization with high density EEG: how many electrodes are needed? Clin Neurophysiol 2003;114:63-9. 
Lorenz J, Hauck M, Paur RC, Nakamura Y, Zimmermann R, Bromm $B$, et al. Cortical correlates of false expectations during pain intensity judgments-a possible manifestation of placebo/nocebo cognitions. Brain Behav Immun 2005;19:283-95.

Macaluso E, Driver J. Multimodal spatial representations in the human parietal cortex: evidence from functional imaging. Adv Neurol 2003;93:219-33.

Mazziotta J, Toga A, Evans A, Fox P, Lancaster J, Zilles K, et al. A four-dimensional probabilistic atlas of the human brain. J Am Med Inform Assoc 2001;8:401-30.

McCaul KD, Haugtvedt C. Attention, distraction, and cold-pressor pain. J Pers Soc Psychol 1982;43:154-62.

McCracken LM. Contextual cognitive behavioral therapy for chronic pain 2005; vol. 33. IASP Press; 2005.

Michael ES, Burns JW. Catastrophizing and pain sensitivity among chronic pain patients: moderating effects of sensory and affect focus. Ann Behav Med 2004;27:185-94.

Michel CM, Lantz G, Spinelli L, De Peralta RG, Landis T, Seeck M. 128-channel EEG source imaging in epilepsy: clinical yield and localization precision. J Clin Neurophysiol 2004;21:71-83.

Morley S, Shapiro DA, Biggs J. Developing a treatment manual for attention management in chronic pain. Cogn Behav Ther 2004;33:1-11.

Mulert C, Jager L, Schmitt R, Bussfeld P, Pogarell O, Moller HJ, Juckel G, Hegerl U. Integration of fMRI and simultaneous EEG: towards a comprehensive understanding of localization and timecourse of brain activity in target detection. Neuroimage 2004;22:83-94.

Nagai Y, Critchley HD, Featherstone E, Fenwick PB, Trimble MR, Dolan RJ. Brain activity relating to the contingent negative variation: an fMRI investigation. Neuroimage 2004;21:1232-41.

Nichols TE, Holmes AP. Nonparametric permutation tests for functional neuroimaging: a primer with examples. Hum Brain Mapp 2002;15:1-25.

Okuda J, Fujii T, Ohtake H, Tsukiura T, Tanji K, Suzuki K, et al. Thinking of the future and past: the roles of the frontal pole and the medial temporal lobes. Neuroimage 2003;19:1369-80.

Pascual-Marqui RD, Esslen M, Kochi K, Lehmann D. Functional imaging with low-resolution brain electromagnetic tomography (LORETA): a review. Methods Find Exp Clin Pharmacol 2002;24:91-5.

Peyron R, Garcia-Larrea L, Gregoire MC, Costes N, Convers P, Lavenne $\mathrm{F}$, et al. Haemodynamic brain responses to acute pain in humans: sensory and attentional networks. Brain 1999;122:1765-80.

Ploghaus A, Becerra L, Borras C, Borsook D. Neural circuitry underlying pain modulation: expectation, hypnosis, placebo. Trends Cogn Sci 2003;7:197-200.

Ploghaus A, Tracey I, Gati JS, Clare S, Menon RS, Matthews PM, et al. Dissociating pain from its anticipation in the human brain. Science 1999;284:1979-81.

Porro CA, Baraldi P, Pagnoni G, Serafini M, Facchin P, Maieron M, et al. Does anticipation of pain affect cortical nociceptive systems? J Neurosci 2002;22:3206-14.

Porro CA, Cettolo V, Francescato MP, Baraldi P. Functional activity mapping of the mesial hemispheric wall during anticipation of pain. Neuroimage 2003;19:1738-47.
Posner MI, Petersen SE. The attention system of the human brain. Annu Rev Neurosci 1990;13:25-42.

Price DD. Psychological mechanisms of pain and analgesia 1999; vol. 15. IASP Press; 1999

Rao RP, Ballard DH. Predictive coding in the visual cortex: a functional interpretation of some extra-classical receptive-field effects. Nat Neurosci 1999;2:79-87.

Rao RPN, Olshausen BA, Lewicki MS. Probabilistic models of the brain: perception and neural function. MIT Press; 2002.

Seeck M, Lazeyras F, Michel CM, Blanke O, Gericke CA, Ives J, Delavelle J, Golay X, Haenggeli CA, de Tribolet N, Landis T. Non-invasive epileptic focus localization using EEG-triggered functional MRI and electromagnetic tomography. Electroencephalogr Clin Neurophysiol 1998;106:508-12.

Semlitsch HV, Anderer P, Schuster P, Presslich O. A solution for reliable and valid reduction of ocular artifacts, applied to the P300 ERP. Psychophysiology 1986;23:695-703.

Seymour B, O'Doherty JP, Dayan P, Koltzenburg M, Jones AK, Dolan RJ, et al. Temporal difference models describe higher-order learning in humans. Nature 2004;429:664-7.

Towle VL, Bolanos J, Suarez D, Tan K, Grzeszczuk R, Levin DN, et al. The spatial location of EEG electrodes: locating the bestfitting sphere relative to cortical anatomy. Electroencephalogr Clin Neurophysiol 1993;86:1-6.

Trebuchon-Da Fonseca A, Giraud K, Badier JM, Chauvel P, LiegeoisChauvel C. Hemispheric lateralization of voice onset time (VOT) comparison between depth and scalp EEG recordings. Neuroimage 2005;27:1-14.

Vase L, Robinson ME, Verne GN, Price DD. Increased placebo analgesia over time in irritable bowel syndrome (IBS) patients is associated with desire and expectation but not endogenous opioid mechanisms. Pain 2005;115:338-47.

Vitacco D, Brandeis D, Pascual-Marqui R, Martin E. Correspondence of event-related potential tomography and functional magnetic resonance imaging during language processing. Hum Brain Mapp 2002;17:4-12.

Vlaeyen JW, Linton SJ. Fear-avoidance and its consequences in chronic musculoskeletal pain: a state of the art. Pain 2000;85:317-32.

Wager TD. Expectations and anxiety as mediators of placebo effects in pain. Pain 2005;115:225-6.

Wager TD, Rilling JK, Smith EE, Sokolik A, Casey KL, Davidson RJ, et al. Placebo-induced changes in FMRI in the anticipation and experience of pain. Science 2004;303:1162-7.

Watson A, El-Deredy W, Vogt B, Jones AKP. Categories of placebo response in the absence of site-specific expectation of analgesia. Pain 2006;126:115-22.

Wiggs CL, Weisberg J, Martin A. Neural correlates of semantic and episodic memory retrieval. Neuropsychologia 1999;37:103-18.

Yao J, Dewald JP. Evaluation of different cortical source localization methods using simulated and experimental EEG data. Neuroimage 2005;25:369-82.

$\mathrm{Yu}$ AJ, Dayan P. Uncertainty, neuromodulation, and attention. Neuron 2005;46:681-92.

Zumsteg D, Friedman A, Wennberg RA, Wieser HG. Source localization of mesial temporal interictal epileptiform discharges: correlation with intracranial foramen ovale electrode recordings. Clin Neurophysiol 2005;116:2810-8. 